\title{
Téoros
}

Revue de recherche en tourisme

\section{Les leçons de la consultation de Parcs Canada dans les Rocheuses}

\section{Normand Cazelais}

Volume 14, numéro 1, printemps 1995

Les parcs : des réseaux en mutation

URI : https://id.erudit.org/iderudit/1077058ar

DOI : https://doi.org/10.7202/1077058ar

Aller au sommaire du numéro

Éditeur(s)

Université du Québec à Montréal

ISSN

0712-8657 (imprimé)

1923-2705 (numérique)

Découvrir la revue

Citer cet article

Cazelais, N. (1995). Les leçons de la consultation de Parcs Canada dans les

Rocheuses. Téoros, 14(1), 50-51. https://doi.org/10.7202/1077058ar d'utilisation que vous pouvez consulter en ligne.

https://apropos.erudit.org/fr/usagers/politique-dutilisation/ 


\title{
Les leçons de la consultation de Parcs Canada dans les Rocheuses
}

\author{
Normand Cazelais ${ }^{\circ}$
}

* Le tourisme et les expériences vécues dan les paros sont eventiels à la réalisation du mandat fondamental des pares nationaux, $m$

Cette assertion fait partie des commentaires de Parcs Canada récemment publiés dans le deuxième bulletin ${ }^{(1)}$ consacré à l'examen des plans directeurs des parcs Banff, Jasper, Kootenay et Yoho dans les Rocheuses. Ce bulletin fait état de la consultation publique menée l'été dernier sur cette question.

Aux yeux de l'organisme fédéral, la participationa étéjusqu'ici «très encourageante»: distribution à 10000 exemplaires du premier bulletin d'information; assistance de quelque 1200 personnes aux journées «portes ouvertes»; tenue de plusieurs rencontres officielles et «non officielless; réception de plus de 2500 communications écrites; nombreuses demandes d'informations supplémentaires.

Les médias ont relevé, dans leurs fréquents reportages, que la révision de la gestion de ces parcs a fait ressortir de nombreuses interrogationset un ensemble d'enjeux qui, d'une part, dépassent les limites des quatre parcs et qui, d'autre part, touchent les relations entre le tourisme et les parcs naturels. Au fond, davantage est mis en cause: il s'agit de la conception même des parcs nord-américains et de l'insertion de lhomme dans la nature.

A cet effet, il est utile de rappeler le contexte de l'apparition des grands parcs au Canada et aux Erats-Unis: sous la pression de la conquête d'un Nouveau Monde où tout était devenu une ressource à consommer sinon à sacrifier au bénéfice le plus immédiat des activités humaines, il était devenu impérieux de protéger des milieux naturels particulièrement beaux, rares ou sensibles en créant des périmètres d'exclusion. Il fallait défendre la nature contre les hommes: de cette préoccupation, alliée à une certaine conception puritaine du

Monsieur Normand Cazelais est chroniqueur en tourisme et conseiler a la vice-présidence-Envronnement de I"Hydro-Québec. mondeetcaractéristiquedel'universanglosaxon nord-américain, a émergécette forme de pensée, dans la conception et la gestion des parcs naturels, que l'homme, en quelque sorte, ne fait pas vraiment partie de la nature. Elle s'est aussi exprimée par cette volonté initiale et persistante d'aménager des parcs *naturels où seraient absentes lesimplantationsetinstallations humaines: dans cet esprit, imaginer et donner naissance à un parc a toujours impliqué d'exproprier les personnes pouvant demeurer de façon permanente ou sporadique sur les territoires considérés.

Cette conception est fort loin de la conception européenne des parcs enaturels: où, au contraire, la présencedes hommes et l'exercice de certaines de leurs activités sont recherchés et favorisés; pensons, entre autres, à New Forest (qui a tout de même plus de neuf cents ans...) dans le sud de l'Angleterre ou au parc de la Corse (Parc national régional) où de grands efforts furent déployés encorerécemment pour faire revivredesvillages etredynamiser l'élevage en milieu montagnard.

Dans le cas des quatre parcs susnommés, la consultation a fait ressortir deux positions qui vont à la fois dans le sens et à l'encontre d'une telle vision :

- l'une, de Parcs Canada - et probablement de l'appareil gouvernemental —, est portée à reconsidérer, pour de multiples raisons tant politiques qu'économiques ou même philosophiques ou administratives, plusieurs aspects de cette exclusion humaine;

- l'autre, du public-du moins de celui qui s'est exprimé, sans qu'on sache réellement, vu les moyens employés, sil est représentatif de l'ensemble de la population - semble confirmer cette volonté d'exclusion.

A ce sujet, dans l'avant-propos du bulletin, la firme Praxis, chargéede mettreenoeuvre la consultation et d'en analyser les résultats, souligne limportance de la question de l'aménagement dans ces quatre parcs. Trois personnes contre une s'opposent à tout aménagement future. Ceux qui sont en faveur allèguent le tourisme et le développement.

\section{* Si beancoup de gens ont rappelé à Parcs Canada que lesparon'existent pas seulenent pour la faune et la flore, il semble que ce soit pour une question d'accès plutôt que dionénagement,s}

Dans ce bulletin, Parcs Canada souligne avoir entreprisl'examendes plansdirecteurs actuels des parcs Banff, Jasper, Kootonay et Yoho, achevés en 1988 жau terme d'un grand programmepublicdeplanification $\%$, pour répondre aux Principes directewr et politiques de gestion de Parc Canada (adoptés en 1994) et aux exigences de la Loi sur les parcs nationas. qui stipule qu'ils doivent être revus tous les cinq ans.

Ces quatreparcs, on le sait, ont étédéclarés Site du patrimoine mondial. Leur sbeauté naturelleen a faitune destination touristique de choix pour des millions de visiteurs qui s'y rendent chaque année de partout dans le mondes. C'est pourquoi, juge Parcs Canada, il жest essentiel de les gérer avec prudence, en s'assurant que le public en profite sans mettre en péril leur intégrité écologiqueà long terme, pour maintenir ce patrimoine montagnard $\%$.

La consultation a permis d'aborder plusieurs sujets (l'intégration régionale; une vision globale pour les quatre parcs; les restrictions budgétaires; l'accès depuis les terres adjacentes; la sécurité du public, les revendicationsterritoriales autochtones, la gestion de l'arrière-pays, la fermeture de certaines zones et installations; etc.) qu'il serait trop long d'analyser ici. Portons par contre un oell attentif à des questions touchant d'abord ces parcs en particulier et ensuite des enjeux de portée générale.

\section{Quelques enjeux particuliers}

Dans le cas de Banffet Yoho, Parcs Canada propose d'interdire aux véhicules moteurs la route $1 \mathrm{~A}$ à l'ouest de Lake Louise et de la convertir en piste de vélo et de randonnée 
pédestre, invoquant sa faible utilisation et les coûts que nécessiterait sa réfection, position qu'appuient les répondants, dans une proportion de quatre contre un.

Au parc Kootenay, Parcs Canada entend permettreaux pourvoyeursetàleursclients ed'utiliser les sentiers del'arrière-pays pour accéder aux terres provinciales environnantess, ce à quoi s'opposent la majoritédespersonnesetorganismesayant exprimé un avis sur la question: le parc, disent-ils, «ne doit pas être utilisé pour l'accès aux territoires de chasse et aux parcs provinciaux adjacentss, tout comme «doit être interdit le transport des armes à feu et du gibier».

De même, selon tous les répondants ou presque, Parcs Canada *ne doit prévoir aucune installation* nouvelle ou supplémentaire à Kootenay, pour les sgroupes commerciaux ou pour satisfaire une plus forte fréquentation d'autocars.

Au parc Banff, l'avenir de l'agglomération de Lake Louise pose de multiples interrogations. En 1979, Parcs Canada a produit à son sujet un plan d'action qui planifiait une faible croissance; l'«avant-projet de mise à jour» confirme son maintien *en tant quecentre de services pour les visiteurs, tout en limitant de manière stricte la croissance commerciale et résidentielles.

Cepland'action a suscité «énormément de commentairess. Les opinions favorables et défavorables à l'échange de terrains concernant le West Louise Lodge (Wapta Lodge) sont, entre autres, nez à nez; pour certains, une proposition d'échange ne seraitacceptableque ssielle estavantageuse au plan de l'environnements.

Par ailleurs, les avis «qui appuient au plus hautpoint la politiqueactuelledecroissance nulle de Lake Louise sont plus nombreux que ceux qui «favorisent la réouverture du dossier [...] en vue de permettre d'autres projets d'aménagementw, y compris l'échange de terrains pour le West Louise Lodge, l'amélioration des installations de ski alpin et la construction d'un centre de congrès au Château Lake Louise.

Dans ses commentaires, Parcs Canada rappelle que l'agglomération fait partie de la région étudiée par un groupe de travail chargé de se pencher sur la vallée de la Bow (a Banff); son mandat est de l'analyser depuis l'entrée est du parc jusqu'à l'amont du bassin versant. L'organisme fédéral entend *reporter indéfiniment toure autre activité liée au plan d'action relatif à Lake Louises pour permettre à cette équipe de terminer son travail et de déposer son rapport (prévu en 1996); «aucune modification au plan d'affectation des terres ne sera permise» d'ici là.

\section{Enjeux généraux}

\section{*La gestion en fonction des écosystèmes:}

Ce mode de gestion «met l'accent sur les liens entre les éléments de la nature et l'importance de l'êtrehumain comme partie de ces éléments. Il constitue «un des thèmes, les plus importants sur lesquels Parcs Canada voulait obtenir des commentaires, et que la plupart des Canadiens ne connaissaient pas\%. Si les répondants l'ont «appuyé en très grande majorité, un grand nombre ont aussi cru nécessaire de rappeler à Parcs Canada que les êtres humains y ont autant leur place que la faune et la flores.

Dans sa réponse, Parcs Canada écrit notamment: *L'être humain fait partie de l'écosystème. Dans un système structuré en fonction del'environnement, l'influence de l'homme varie d'une zone à l'autre. Il domine dans les villeset les centresd'accueil des visiteurs, tandis que la nature domine dans les milieux sauvages. L'influence de l'être humain est un facteur que l'on doit toujoursconsidérer en prenant desdécisions qui touchent l'écosystème».

\section{*Le tourisme durablew}

Le tourisme, on l'a vu, représente un enjeu d'envergure aux yeux de Parcs Canada qui a présenté, lors de la consultation, un document de travail sur une stratégie définissant son rôle dans ces quatre parcs des Rocheuses.

$$
\text { * D'après la majorité des }
$$
commentaires, le public veut lassurance que Pans Canada adopte comme priorites de protéger l'environnement, dassumer lintégrité de l'écosystime et d'interdire ou, du moins, de restreindre de beaucoutp l'aménagenent d'infrastructures comme les centres commerciaux, les terrains de golf, Les centres deski, lespiscines, etc. [...]
Parcs Canada devrait encourager "th écotourisme responsable, durable et à faible impact.»

Pour Parcs Canada, le tourismeet les expériences vécues dans les parcs sont, on l'a vu, «essentiels à la réalisation du mandat fondamental des parcs nationaux». S'appuyant sur les fruits de la consultation, Pares $\mathrm{Ca}$ nada compte done développer une stratégie articulée selon les principes suivants:

- élaboration de pratiques de réglementation et de gestion assurant «la stabilité et la compétitivitéà long terme de l'industries;

- mise au point de produits et de services «fondés sur les valeurs patrimoniales des parcs, surtout en termes d'authenticité, de qualité et de durabilités;

- mesure, sous forme d'études de marché, des «avantages et effets sociaux et économiques du tourisme patrimonial»;

- développement dans le public d'une *meilleure connaissance des parcs».

Il serait certes très intéressant de scruter dansledétail tousces avisafin d'en connaître les particularités et les nuances, afin devoir également à quel pointvivent des positions opposées et quels sont leurs appuis véritables en termes de nombre.

Le résumé établi par Parcs Canada et la firme Praxis laisse comprendre que, sur bien des points, s'affrontent deux écoles de pensée. Yaura-t-il d'ailleurs affrontement, une fois la révision des plans directeurs des quatre parcs? Parcs Canada aura à résoudre une forme de quadrature du cercle s'il veut satisfaire tout le monde et son père. Mais, avant toute chose, avant surtout de déposer des plans d'action et des stratégies, ildevra présenter et défendre clairement sa vision des parcs naturels et de la place que l'homme occupe dans la nature ${ }^{(2)}, f$

\section{NOTES}

(1) Les quatre pares des Rocheuses - Mise à jour quinquennale - Compte rendu sommaire de la participation du public et commentaires de Parcs Canada, Patrimoine canadien/Parcs Canada, non dat $\hat{\theta}, 26$ pages et un avant-propos.

(2) La mise à jour des plans directeurs des quatre parcs des Rocheuses sera disponible, assure Parcs Canada, au printemps 1995. Le prochain examen des plans directeurs aura lieu en 1999 et P'on prévoit qu'il aura une aportée plus globale que ta présente mise aै jour $\mathrm{s}$. 